\title{
COMUNICACIÓN
}

\section{NIVELES CRÍTICOS DE AFLATOXINA EN MUESTRAS DE MAÍz PARA CONSUMO ANIMAL EN LIMA METROPOLITANA}

\author{
Juan Caballero M. ${ }^{1}$, Teresa Arbaiza F. ${ }^{2}$ y Orlando Lucas A. ${ }^{2}$
}

\section{Aflatoxin contamination of yellow corn in the Metropolitan Lima animal feed supply}

\begin{abstract}
A total of 50 samples of hard yellow corn (Zea mays), purchased at the principal wholesale markets of Lima and Callo, were subjected to ELISA test in order to determine the presence of afalotoxins. Contamination $(B 1+B 2+G 1+G 2)$ was found in $82 \%$ of the samples, with concentrations averaging $4.2 \mathrm{ppb}$ (from 0 to $37.8 \mathrm{ppb}$ ). The samples of Peruvian grown yellow corn had a $95 \%$ contamination rate, with concentrations from 0 to $37.8 \mathrm{ppb}$, and $20 \%$ of the samples exceeding permissible levels (10 ppb) of the Codex Alimentarius. In contrast, the imported corn had concentrations from 0 to $12.5 \mathrm{ppb}$, with only $3.3 \%$ of the samples exceeding permissible levels. These results document important, illness causing, aflatoxin contamination of the metropolitan Lima animal feed supply.
\end{abstract}

Key words: Aflatoxins, Aflatoxicosis, Mycotoxicosis, Zea mays.

Palabras clave: Aflatoxinas, Aflatoxicosis,Micotoxicosis, Zea mays.

Las aflatoxinas son potentes agentes carcinógenos, mutágenos, teratógenos y capaces de provocar lesiones hepáticas en diversas especies animales, pudiendo causar tumores hepáticos. La propiedad carcinógena es la de mayor importancia en los aspectos de sanidad de los alimentos (Basilico, 1995).

Las aflatoxinas son conocidas desde 1960, cuando en Inglaterra se presentó una epidemia que mató alrededor de 100,000 pavipollos, patitos y pollos con una pérdida de cientos de miles de dólares. La causa de esta enfermedad, presumiblemente se debía a un contaminante del alimento destinado al consumo animal, probándose que tanto la toxigenicidad y la carcinogenicidad ocasionada en los animales se debió a la contaminación con el hongo Aspergillus flavus.
En el Perú, según reportes del Ministerio de Agricultura, se importan grandes volúmenes de maíz amarillo destinados a la elaboración de alimentos balanceados para animales (Ministerio de agricultura, 1998). Si bien es cierto que los importadores realizan análisis de los productos que compran, los resultados no son reportados, ni publicados.

El maíz es el componente básico en la formulación de la mayoría de los productos balanceados destinados a los animales de granja y de compañía, por lo que se plantea este estudio para conocer los niveles de aflatoxina presentes en el maíz amarillo importado y de cosecha nacional (refinado, partido y entero) y determinar la correlación entre la humedad y el nivel de aflatoxinas, de tal manera que esa información puede ser utilizada por los ganaderos, criadores y autoridades del sector pecuario.

\footnotetext{
1 Práctica privada

${ }^{2}$ Laboratorio de Nutrición, FMV-UNMSM
} 


\section{J. Caballero et al.}

Se tomaron al azar 50 muestras de maíz amarillo (Zea maiz) variedad indurata y de origen importado (30) y nacional (20).

Las muestras de maíz importado se obtuvieron directamente de los barcos cargueros, acodados a dos millas del terminal marítimo del Callao, utilizando un muestreador de cereales. Se muestreó barcos provenientes de Estados Unidos y Argentina, países de los que más volumen de maíz se importa. La cantidad de muestra por barco fue de $10 \mathrm{Kg}$.

Las muestras de maíz amarillo nacional se obtuvieron en los mercados mayoristas de Lima Metropolitana bajo la forma de maíz entero, partido y refinado.

En el laboratorio, se procedió a reducir el tamaño de las muestras, mediante la técnica del cuarteo, hasta obtener una submuestra de $200 \mathrm{~g}$ aproximadamente, determinándose la humedad en 5 a $6 \mathrm{~g} \mathrm{y}$ aflatoxina en $50 \mathrm{~g}$; el excedente se guardó en el congelador como contra muestra (Arbaiza, 1998).

Para la determinación del contenido de Aflatoxinas se utilizó el kit Veratox AST (ELISA competitivo directo) para la determinación de aflatoxinas totales $\left(\mathrm{B}_{1}+\mathrm{B}_{2}+\right.$ $G_{1}+G_{2}$ ), que consiste en extraer las aflatoxinas de la muestra molida con una solución de metanol al 70\%, mediante licuado y filtración.
La intensidad del color rojo es proporcional a la cantidad de toxina presente. El color azul indica que las muestras son negativas. Las absorbancias de muestras y controles se midieron en el lector de ELISA BIOTEK EL301 a una longitud de onda de $650 \mathrm{~nm}$. Los cálculos se expresan en partes por billón (ppb) que equivale a $\mu \mathrm{g} / \mathrm{kg}$.

Los resultados de los niveles de aflatoxinas de las muestras de maíz nacional e importado se muestran en el Cuadro 1 , se observa que el maíz nacional tiene mayor nivel de contaminación con aflatoxinas que el maíz importado.

En cuanto al maíz de cosecha nacional, las cuales fueron agrupadas en: maíz refinado, maíz partido y maíz entero, se observa que de los tres el maíz refinado tiene el mayor nivel de contaminación (Cuadro 2).

La humedad varió desde $9.3 \%$ hasta $12.6 \%$ con un promedio de $10.98 \%$. Según al análisis de regresión lineal no se encontró correlación $(p<0.05)$ entre los niveles de aflatoxinas y la humedad de las muestras de maíz.

De acuerdo a los resultados obtenidos, los porcentajes de humedad no están asociados a los niveles de aflatoxinas. El promedio de humedad de las muestras de maíz fue $10.98 \%$. Al respecto el Instituto de Investigación Tecnológica y de Normas Técnicas (ITINTEC) del Perú establece una humedad

Cuadro 1. Resultado de los niveles de aflatoxinas (ppb) en muestras de maíz según procedencia

\begin{tabular}{lccc}
\hline & Nacional & Importado & Total \\
\hline Media & 5.95 & 2.65 & 3.96 \\
Desviación estándar & 8.45 & 2.82 & 5.92 \\
Rango & $0-37.8$ & $0-12.5$ & $0-37.8$ \\
Muestras no aptas * & $20 \%$ & $3.3 \%$ & $10 \%$ \\
\hline
\end{tabular}

*: nivel de aflatoxinas superior al nivel permisible por el Codex Alimentarius $(10 \mathrm{ppb})$ 
Cuadro 2. Resultado de los niveles de aflatoxinas (ppb) en tres tipos de maíz nacional.

\begin{tabular}{lccc}
\hline & \multicolumn{3}{c}{ Maíz } \\
\hline & Refinado & Partido & Entero \\
\hline Media & 9.83 & 4.27 & 2.93 \\
Desviación estándar & 13.33 & 3.87 & 0.81 \\
Rango & $0-37.8$ & $0.7-14.2$ & $1.8-3.7$ \\
Muestras no aptas $^{\text {a }}$ & $42 \%$ & $11.1 \%$ & $0 \%$ \\
\hline
\end{tabular}

a: nivel de aflatoxinas superior al nivel permisible por el Codex Alimentarius $(10 \mathrm{ppb})$.

máxima de $14.5 \%$ en el maíz amarillo duro (variedad indurata).

El alto nivel de contaminación por aflatoxinas en las muestras de maíz nacional ( $20 \%$ de las muestras no aptas según el nivel máximo establecido por el Codex Alimentarius), es un indicativo que el nivel de contaminación en nuestro maíz es alto, a pesar de que morfológicamente es un maíz mejor presentado, con color, tamaño y forma muy superior al importado, aunque en algunas muestras sobre todo en las refinadas se percibía un color y olor desagradable.

El máximo nivel de aflatoxinas del maíz importado fue $12,5 \mathrm{ppb}$, lo que concuerda con los niveles encontrados en un monitoreo realizado durante 6 años en Estados Unidos, en donde se detectó niveles entre 5-20 $\mu \mathrm{g} / \mathrm{kg}$ (Park, 1993) lo que reafirma los resultados obtenidos en este trabajo.

El nivel máximo para alimento animal, establecido por la Food and Drug Administration (FDA) de los Estados Unidos es 20 ppb (Park, 1993), de acuerdo a esto, sólo el nivel de aflatoxinas de una de las $\mathbf{5 0}$ muestras de maíz analizadas, fue superior a este nivel.

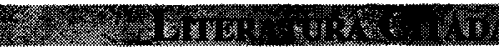

1. Arbaiza, F.T. 1998. Riesgo De La Contaminación Con Aflatoxina en Maní (Arachis Hipogaea) de Producción Nacional. Tesis de Magister, Facultad de Farmacia y Bioquímica, UNMSM, pp. 89.

2. Basilico, J.C. 1995. Micotoxínas en Alimentos. El Riesgo Sobre La Mesa, Centro de Publicaciones U.N.L. Santa Fe, Argentina, p. 28-42 .

3. Ministerio de Agricultura. 1998. Producción Hortofrutícola, p. 345. LimaPerú.

4. Park, D.L 1993. Controlling Aflatoxin In Food And Feed. Food Technol., Vol. 10, p.92-96. 